\title{
FORZA ITALIA A LIVELLO LOCALE: UN MARCHIO IN FRANCHISING?
}

\author{
di Caterina Paolucci
}

In questo saggio presenterò alcuni risultati di una ricerca empirica sugli amministratori locali di Forza Italia, che si inserisce in un più ampio progetto di analisi del processo di istituzionalizzazione di questo partito ${ }^{1}$. La scelta di concentrare l'attenzione sugli eletti locali nasce dalla convinzione che il futuro di Forza Italia sia legato non solo agli eventi organizzativi e politici che la riguardano a livello nazionale e di leadership, ma anche allo sviluppo di strutture e procedure preposte alla formazione e al consolidamento di una classe dirigente locale. In altri termini, la persistenza organizzativa di questo nuovo partito dipenderà dalla realizzazione, quantomeno a livello locale, di quella «infusione di valori» che rappresenta la premessa indispensabile di ogni compiuta istituzionalizzazione (Huntington 1968, 15; Selznick 1957, 21-22). Il fatto che a cinque anni dalla sua fondazione Forza Italia sia ancora un'organizzazione patrimoniale fortemente caratterizzata in senso personalistico ed aziendalista (Maraffi 1996; Panebianco 1995), suggerisce che tale infusione di valori, e dunque l'inizio del processo di consolidamento organizzativo, non possa aver luogo per impulso delle élites nazionali. A livello centrale questo processo è reso infatti assai difficile dalle modalità di reclutamento e selezione del personale partitico e parlamentare, miranti a creare o confermare un rapporto di fiducia personale con il leader Silvio Berlusconi, tale per cui la legittimazione dei prescelti diventa una funzione diretta delle oscillazioni del rapporto fiduciario che li lega al capo. È a quest'ultimo, non già all'organizzazione, che si

1 Il saggio presenta una parte dei dati di un sondaggio postale degli amministratori locali di Forza Italia condotto nell'aprile-settembre 1997, realizzato grazie a un finanziamento del Consiglio Nazionale delle Ricerche nell'ambito del progetto «Partiti e sistemi di partito in Italia. Le trasformazioni organizzative» diretto da Leonardo Morlino.

RIVISTA ITALIANA DI SCIENZA POLITICA / a. XXIX, n. 3, dicembre 1999 
dirige la lealtà delle élites centrali, e non potrebbe essere altrimenti, data la natura del partito. A livello locale, al contrario, la distanza dal centro potrebbe aver favorito un reclutamento più aperto e una selezione meno personalistica, e dunque l'emergere di un personale politico più libero di dirigere la propria lealtà verso l'organizzazione in sé, piuttosto che esclusivamente verso il suo capo. Pertanto, se esiste una possibilità di consolidamento per Forza Italia, essa è sicuramente maggiore a livello locale. In questa prospettiva è necessario dunque valutarne le dinamiche organizzative sul territorio, tenendo presente tuttavia che l'istituzionalizzazione presuppone l'esistenza di un legame tra centro e periferia che favorisca coesione e coordinamento tra i diversi livelli, senza le quali difficilmente l'organizzazione potrà stabilizzarsi nel tempo (Huntington 1968, 23).

Ai fini di questo studio del processo di istituzionalizzazione abbiamo deciso inoltre di concentrarci in modo particolare sugli eletti nelle amministrazioni locali, trattando soltanto in via indiretta $i$ dirigenti locali del partito. Ciò per una ragione analoga a quella che ci ha indotto a indirizzare lo studio sul livello locale invece che sul livello nazionale di Forza Italia: la maggior parte dei dirigenti periferici sono nominati a cascata a partire dal vertice nazionale, e dunque risultano legati ad esso da un rapporto fiduciario, al pari dei parlamentari e dei dirigenti nazionali (Hopkin e Paolucci 1999). Essi non possono pertanto rappresentare una forza in grado di emancipare il partito da quella logica patrimoniale che già ne rende difficile l'istituzionalizzazione a livello centrale. Inoltre, la concentrazione del potere organizzativo nella figura del coordinatore regionale, nominato direttamente da Berlusconi, e la scarsità di risorse disponibili alle articolazioni territoriali sembrano averne inibito fino ad ora qualsiasi possibilità di autonomizzazione dal centro (Porro 1995). Abbiamo pertanto ipotizzato che solo gli eletti locali sarebbero stati eventualmente in grado di sviluppare un interesse più stabile e autonomo alla sopravvivenza dell'organizzazione, in quanto la loro lealtà nei suoi confronti non sarebbe mediata dalla fedeltà al capo, o lo sarebbe in maniera tanto più blanda quanto più distanti essi si trovano dal nucleo centrale da cui promana l'autorità patrimoniale (Weber 1995, 110). Ma attraverso quali vie si sarebbe potuta sviluppare questa identificazione organizzativa da parte dei rappresentanti periferici, e sarebbe stato possibile un consolidamento di tale legame senza un forte input da parte del centro? 
In un partito relativamente nuovo come Forza Italia l'interesse alla sopravvivenza dell'organizzazione può iniziare a svilupparsi solo se si realizza una complementarità tra le motivazioni individuali dei partecipanti, in questo caso gli amministratori locali, e gli incentivi offerti loro dall'organizzazione (Sorauf 1968, 92; Clark e Wilson 1961, 134-5). Obiettivo di questo saggio è mostrare se tale complementarità si sia realizzata. I quesiti con i quali occorrerà confrontarsi sono quelli che fanno emergere le specificità del rapporto di scambio tra eletti e organizzazione partitica. In particolare si cercherà di capire se e quali incentivi vengano offerti dal partito ai suoi eletti per garantirne la partecipazione, e se gli eletti ritengano o meno soddisfatte, attraverso tali incentivi, le motivazioni individuali che li hanno inizialmente indotti a partecipare. Questa analisi ci consentirà di valutare l'efficacia organizzativa di Forza Italia a livello locale, e di avanzare delle ipotesi sulle sue prospettive di istituzionalizzazione.

Dopo una breve esposizione della base empirica della ricer$\mathrm{ca}$, questo articolo si snoderà lungo un percorso che segue alcune delle tappe principali dell'instaurazione del rapporto di scambio tra il partito e i suoi eletti:

- Il reclutamento e la selezione dei candidati. Questa prima tappa fornisce un importante quadro d'insieme da cui partire per individuare le potenzialità di istituzionalizzazione di un partito politico. Il modello di reclutamento, centralizzato e gerarchico oppure decentrato e laterale influenza in misura consistente il grado di autonomia e la coerenza organizzativa. Inoltre, le caratteristiche dei selezionati sono indicative delle aspettative dei selezionatori, ma anche di quelle degli eletti nei confronti dell'organizzazione (Roback 1974, 22; Norris 1997, 15).

- La campagna elettorale. L'evento elettorale rappresenta un momento cruciale per il futuro organizzativo di un partito, in quanto fissa le caratteristiche dello scambio tra incentivi (materiali o di solidarietà) e partecipazione (candidatura sotto la bandiera del partito). In questa fase dovrebbe consolidarsi l'autonomia dell'organizzazione, e nel contempo la sua unità, attraverso lo sviluppo delle capacità di coordinamento e perseguimento dell'interesse collettivo (Huntington 1968, 23).

- Il dopo elezioni e l'integrazione dell'eletto nell'organizzazione. La fase più lunga e stabile nel rapporto tra eletto e partito è quella che si snoda tra un'elezione e la successiva, e che vede l'amministratore inserito nelle istituzioni locali e al con- 
tempo membro del partito. Le questioni che esploreremo sono relative al livello di integrazione degli eletti nelle strutture e al loro grado di soddisfazione rispetto all'organizzazione - ciò consente di apprezzare l'efficacia organizzativa, soprattutto rispetto alla distribuzione degli incentivi, e rappresenta un buon indicatore dell'avanzamento del processo di infusione di valori e dunque del potenziale di istituzionalizzazione del partito.

\section{La base empirica della ricerca}

L'unità d'analisi della ricerca presentata in parte in questo saggio è costituita dagli eletti nelle amministrazioni locali (regionali, provinciali e comunali) di Forza Italia. Secondo i dati forniti da Forza Italia e dal ministero degli Interni, gli eletti locali di Forza Italia al momento della rilevazione (aprile-settembre 1997) erano 1.815. Per una prima indagine si è deciso di estrarre un campione rappresentativo ammontante a circa un quarto del totale. I criteri che hanno indirizzato il campionamento sono stati la distribuzione per livello amministrativo (regionale, provinciale, comunale), e per area geografica (Nord, Centro e Sud). Si sono effettuati così tre campionamenti distinti per livello amministrativo, realizzati in modo tale da introdurre un bias a favore del gruppo degli eletti nei consigli regionali. Si è deciso infatti di inviare il questionario a tutti i 167 eletti regionali, ritenendo che, data la rilevanza del livello regionale nell'organizzazione di Forza Italia, valesse la pena concentrare l'attenzione su questo sottoinsieme di eletti, che si ipotizzava essere più integrati in quello che risultava altrimenti un sistema organizzativo debolmente strutturato, soprattutto ai livelli inferiori a quello regionale (vedi infra, nota 2). Per lo stesso motivo, da questo gruppo ci aspettavamo anche un maggior successo in termini di redemption dei questionari. Effettivamente, il tasso di risposta di questo sottoinsieme $(44,3 \%)$ è stato superiore alle più ottimistiche previsioni, confermando la più elevata integrazione organizzativa dei suoi membri. Esso è infatti superiore di otto punti percentuali a quello degli eletti provinciali (36\%) ed è più del doppio del tasso di risposta dei comunali $(20,4 \%)$.

I restanti questionari (circa 300) sono stati suddivisi tra eletti provinciali e comunali in una percentuale pari al $20 \%$ dei rispettivi totali. Pertanto, ai provinciali sono stati inviati 97 questionari, uno per ogni provincia in cui Forza Italia era presente. 
TAB. 1. Distribuzione del campione per livello amministrativo raffrontato alla popolazione degli eletti locali

\begin{tabular}{lccccc}
\hline \multirow{2}{*}{$\begin{array}{l}\text { Livello } \\
\text { amm. }\end{array}$} & $\begin{array}{c}\text { Eletti che } \\
\text { hanno risposto }\end{array}$ & \multicolumn{2}{c}{$\begin{array}{c}\text { Eletti totali } \\
\text { di Forza Italia }\end{array}$} & \multirow{2}{*}{$\begin{array}{c}\text { Differenza percentuale tra } \\
\text { campione e totale eletti }\end{array}$} \\
\cline { 2 - 4 } & $\mathrm{N}$ & $\%$ & $\mathrm{~N}$ & $\%$ & \\
\hline Regionale & 74 & 46,2 & 167 & 14,1 & 31,9 \\
Provinciale & 35 & 21,8 & 467 & 25,7 & $-3,8$ \\
Comunale & 51 & 31,8 & 1183 & 65,0 & $-33,1$ \\
Totale & 160 & 100,0 & 1.815 & 100,0 & \\
\hline
\end{tabular}

Per favorire la redemption gli invii si sono indirizzati ai capogruppo consiliari. In modo totalmente casuale è stato estratto solo il campione dei consiglieri comunali (250 questionari), tenendo ferma la stratificazione territoriale su tre macro aree geografiche (Nord, Centro e Sud) dei comuni superiori a 15.000 abitanti nei quali era presente il gruppo consiliare di Forza Italia (273 comuni).

Dei 514 questionari inviati ne sono rientrati 160, per una redemption complessiva del $31,1 \%$. Tale cifra corrisponde al $7,7 \%$ del totale degli eletti locali di Forza Italia. Un rientro del $31,1 \%$ può essere considerato assai soddisfacente, se confrontato con altre ricerche di questo tipo, considerando la lunghezza del questionario e la complessità delle domande, che richiedevano all'intervistato un considerevole investimento di tempo e di concentrazione.

I consiglieri regionali, come ci aspettavamo e auspicavamo sono nettamente sovra-rappresentati. I provinciali, sono rappresentati in maniera pressocché perfettamente proporzionale, mentre i comunali sono fortemente sotto-rapresentati (tab. 1). $\mathrm{Al}$ bias programmato a favore dei regionali, si aggiunge l'effetto del basso tasso di risposta dei comunali, precostituendo un campione che globalmente sovra-rappresenta i più «integrati» $a$ discapito dei meno integrati nel sistema organizzativo di Forza Italia. Le conclusioni dell'articolo ne risultano pertanto rafforzate, in quanto, se, come vedremo, la coesione organizzativa è risultata bassa tra coloro che sono tutto sommato più integrati nella struttura, si può presumere che il suo livello scenda ulteriormente nei segmenti meno integrati dell'organizzazione. Una indiretta conferma di ciò è stata fornita dai diversi gradi di redemption di ciascun sottoinsieme. 
TAB. 2. Distribuzione del campione per area geografica raffrontato alla popolazione degli eletti locali

\begin{tabular}{lccccc}
\hline \multirow{2}{*}{ Area } & \multicolumn{2}{c}{$\begin{array}{c}\text { Eletti che } \\
\text { hanno risposto }\end{array}$} & \multicolumn{2}{c}{$\begin{array}{c}\text { Eletti totali } \\
\text { di Forza Italia }\end{array}$} & $\begin{array}{c}\text { Differenza percentuale tra } \\
\text { campione e totale eletti }\end{array}$ \\
\cline { 2 - 4 } & $\mathrm{N}$ & $\%$ & $\mathrm{~N}$ & $\%$ & \\
\hline Nord & 50 & 31,3 & 686 & 37,7 & $-6,4$ \\
Centro & 41 & 25,6 & 267 & 14,7 & 10,8 \\
Sud & 69 & 43,1 & 865 & 47,6 & $-4,5$ \\
Totale & 160 & 100,0 & 1.815 & 100,0 & \\
\hline
\end{tabular}

TAB. 3. Distribuzione del campione per regione raffrontato alla popolazione degli eletti locali

\begin{tabular}{lrrrrr}
\hline Regione & \multicolumn{2}{c}{$\begin{array}{c}\text { Eletti che } \\
\text { hanno risposto }\end{array}$} & \multicolumn{2}{c}{$\begin{array}{c}\text { Eletti totali } \\
\text { di Forza Italia }\end{array}$} & $\begin{array}{c}\text { Differenza percentuale tra } \\
\text { campione e totale eletti }\end{array}$ \\
\cline { 2 - 4 } & \multicolumn{1}{r}{ N } & \multicolumn{1}{c}{$\%$} & \multicolumn{1}{c}{$\mathrm{N}$} & $\%$ & \\
\hline Piemonte & 7 & 4,4 & 154 & 8,4 & $-4,0$ \\
Lombardia & 19 & 11,9 & 255 & 14,0 & $-2,1$ \\
Veneto & 15 & 9,4 & 187 & 10,3 & $-0,9$ \\
Friuli & 3 & 1,9 & 34 & 1,8 & 0,1 \\
Liguria & 6 & 3,8 & 51 & 2,8 & 1,0 \\
Emilia-R. & 17 & 10,6 & 95 & 5,2 & 5,4 \\
Toscana & 11 & 6,9 & 95 & 5,2 & 1,7 \\
Umbria & 4 & 2,5 & 21 & 1,1 & 1,4 \\
Marche & 9 & 5,6 & 57 & 3,1 & 2,5 \\
Lazio & 6 & 3,8 & 125 & 6,8 & $-3,8$ \\
Abruzzo & 9 & 5,6 & 44 & 2,4 & 3,2 \\
Molise & 3 & 1,9 & 15 & 0,8 & 1,1 \\
Campania & 8 & 5,0 & 134 & 7,3 & $-2,3$ \\
Puglia & 14 & 8,8 & 176 & 9,6 & $-0,8$ \\
Basilicata & 4 & 2,5 & 18 & 0,9 & 1,6 \\
Calabria & 3 & 1,9 & 40 & 2,2 & $-0,3$ \\
Sicilia & 13 & 8,1 & 251 & 13,8 & $-5,7$ \\
Sardegna & 9 & 5,6 & 62 & 3,4 & 2,2 \\
Totale & 160 & 100,0 & 1.815 & 100,0 & \\
\hline
\end{tabular}

Non dovevano esserci invece distorsioni geografiche, ed effettivamente non ce ne sono di rilevanti (tab. 2). Se si eccettua una leggera sotto-rappresentazione del Nord, dovuta al basso tasso di risposte provenienti dal Piemonte, una sovra-rappresentazione del centro, principalmente dovuta all'alto tasso di risposte provenienti dall'Emilia Romagna, e una sotto-rappresentazione del Sud, imputabile alla bassa redemption degli eletti si- 
ciliani, la distribuzione del campione è in linea con quella della popolazione degli eletti di Forza Italia (tab. 3).

\section{Le modalità di reclutamento e la selezione dei candidati}

Il grado di coesione e autonomia di un partito degli eletti, quale è stato in origine Forza Italia e quale continua ad essere nonostante le modifiche statutarie adottate nel 1997, dipende innanzitutto dal tipo di reclutamento. Da questo primo contatto tra candidato e organizzazione emergono sia le aspettative dei selezionatori verso $\mathrm{i}$ candidati, sia quelle dei futuri eletti nei confronti dell'organizzazione. Un'analisi approfondita dei percorsi di accesso all'attività politica ci consente di iniziare a valutare la reciprocità e compatibilità degli interessi in gioco, preludio quanto meno ad un avvio di istituzionalizzazione.

Nella letteratura sul reclutamento dei candidati alle cariche elettive si distinguono due vie d'accesso idealtipiche alla carriera politica: la via gerarchica o verticale, generalmente impermeabile agli esterni, e la via orizzontale o societaria, permeabile e aperta (Putnam 1976, 47; Norris 1997, 3-5). Le differenze tra questi due percorsi di reclutamento si riflettono sulla coesione organizzativa e sulle possibilità di un'istituzionalizzazione forte. Infatti, un reclutamento di tipo gerarchico contribuisce al rafforzamento della lealtà e, in un partito relativaménte nuovo, può favorire la perdita del carattere strumentale iniziale dell'organizzazione, conducendo ad una professionalizzazione del suo personale. Il reclutamento societario favorisce invece l'accesso alle carriere elettive di dilettanti della politica, la cui lealtà può rivelarsi a tempo determinato, e le cui capacità politiche sono tutte da dimostrare. In questo caso l'istituzionalizzazione non può che essere debole, e dipendere dall'esistenza nel partito di fazioni organizzate in grado di svolgere quella funzione di integrazione verticale, garantita altrove dall'inserimento stabile nella gerarchia del partito.

Salvo alcuni casi particolari, dove era in gioco una forte componente di immagine o interessi locali consolidati, l'attenzione mostrata dalla leadership di Forza Italia per il reclutamento di candidati alle elezioni amministrative è stata molto bassa. In occasione delle elezioni amministrative del 1994 e 1995 Berlusconi esprime dei dubbi sull'opportunità di prendere parte alle contese elettorali, e per un certo periodo pare che Forza Ita- 
lia non si debba presentare con candidati propri. La mobilitazione locale è scarsa e i risultati poco soddisfacenti delle prime tornate locali scoraggiano ulteriormente la partecipazione di base e i tentativi di organizzazione a livello periferico, al punto che Forza Italia si trova in seguito spesso senza aspiranti sufficienti a coprire tutti i posti disponibili. Data la bassa partecipazione locale, anche volendo attingere all'interno delle proprie articolazioni territoriali, non si trova un numero sufficiente di candidati. Insomma, l'offerta di candidature locali è superiore alla domanda, e questo può aver spinto i selezionatori locali ad aprire il reclutamento, rendendolo più permeabile. È ipotizzabile che il disinteresse della leadership per lo sviluppo organizzativo sul territorio e lo squilibrio tra domanda e offerta di candidati abbia reso possibile un tipo di reclutamento meno centralizzato, diverso dal modello patrimoniale prevalente a livello nazionale.

Parlare di reclutamento d'apparato in un partito costituitosi dal nulla nell'arco di pochi mesi, che si dichiara programmaticamente antiburocratico e «leggero», può sembrare incongruo. Tuttavia, abbiamo rivolto una domanda agli eletti relativa ad eventuali ruoli organizzativi o cariche ricoperte in Forza Italia precedentemente all'elezione, ricevendo nel $43,6 \%$ dei casi validi risposte affermative ${ }^{2}$. Si tratta di percorsi che difficilmente si possono denominare «carriere», in quanto sono molto brevi e spesso riguardano ruoli e funzioni veri solo sulla carta, che certo ben poco hanno a che fare con le carriere convenzionali dei professionisti della politica «allevati» all'interno dei partiti tradizionali (McAllister 1997, 17). E tuttavia questa percentuale indica che ove possibile, anche solo per mancanza di candidati, si è cercato di reclutare tra persone già inserite, sebbene da poco, nell'organizzazione. Abbiamo dunque un reclutamento che ove può cerca di sfruttare il canale interno, ma che si rivolge in misura maggiore al mercato esterno. Gli ingressi laterali, e dunque il reclutamento societario, sembrerebbero avere avuto un peso molto consistente anche a livello locale. Ma si è trattato di una scelta o di un percorso obbligato?

Per capire meglio la natura del reclutamento occorre indivi-

2 Alla domanda «Precedentemente alla sua elezione ha ricoperto cariche in Forza Italia?» rispondono affermativamente il $40,6 \%$, negativamente il $52,5 \%$, non risponde il $6,9 \%$. Per quanto riguarda il tipo di carica ricoperta, prevalgono le posizioni a livello provinciale e subprovinciale. 
duarne i soggetti, la direzione e l'obiettivo. Per quanto riguarda i primi due aspetti, bisogna chiarire quali soggetti organizzativi si sono attivati di più nel processo di reclutamento e se il partito si è mosso attivamente contattando, oppure se invece ha «subìto», data la penuria di candidati, l'autocandidatura di chi si è presentato spontaneamente a Forza Italia come aspirante candidato. Soltanto nel primo caso l'esito del reclutamento è centripeto e favorisce il controllo da parte della leadership, e di conseguenza un'istituzionalizzazione forte. Per quanto riguarda invece l'obiettivo del reclutamento, occorre esplorare il processo di selezione, per sapere in che misura a livello locale si è fatto ricorso ad ex professionisti della politica rimasti «disoccupati» in seguito alla virtuale scomparsa o al radicale ridimensionamento di alcuni tra i maggiori partiti della Prima repubblica, e se questi sono stati contattati da Forza Italia o hanno invece cercato di reinserirsi nel gioco politico di propria iniziativa. Inoltre, sarà interessante verificare quanto Forza Italia sia stata in grado di attingere a tutta una serie di professioni da essa programmaticamente «preferite». Le implicazioni delle diverse ipotesi per il futuro organizzativo e il processo di istituzionalizzazione e consolidamento del partito sono molteplici, e le vedremo tra poco.

Il carattere e la composizione dei soggetti del reclutamento, ovvero degli organismi di selezione (selectorates) è fondamentale per due ragioni: in primo luogo il selectorate rappresenta il gruppo (constituency) al quale il prescelto risponde del proprio operato, poiché il suo comportamento è guidato dalla percezione degli interessi, dei desideri e delle richieste di coloro che ne controllano l'accesso alla carica (tenure in office). La seconda ragione è che i selezionatori definiscono le caratteristiche di cui deve essere in possesso l'aspirante candidato (Putnam 1976, 56-57).

Il reclutamento dei nostri intervistati è avvenuto nella metà dei casi attraverso selectorates costituiti da dirigenti inseriti nei ruoli organizzativi locali, con la conseguenza di una gerarchizzazione e potenziale capacità di controllo dell'eletto da parte dell'organizzazione ${ }^{3}$. Nell'altra metà dei casi invece esso ha avuto

3 Il reclutamento è passato in circa metà dei casi attraverso soggetti partitici inseriti nell'organizzazione locale, come il coordinatore regionale e provinciale, il delegato di collegio e il parlamentare del collegio o della circoscrizione. I rappresentanti del partito ai tre livelli in cui si struttura l'organizzazione locale e il parlamentare del luogo si attivano in proporzione molto simile: i coordinatori regionali nel $14,7 \%$ dei casi, i coordinatori provinciali nel $12 \%$, i delegati di collegio nel $9,3 \%$, i parlamentari nel $12 \%$. L'8,5\% degli intervistati inoltre ha avuto contatti con due o più dirigenti locali. 
TAB. 4. Modalità di reclutamento: chi si èlè stato attivato nella fase di reclutamento (\%)

1) dirigente locale /parlamentare di FI

2) amici attivi in FI

3) dirigente nazionale/presidente di club

4) più contatti con esponenti locali di FI

Totale

100,0

luogo attraverso figure nazionali, come i dirigenti centrali, o addirittura extra-gerarchiche, come i presidenti di club e gli «amici attivi nel movimento», con l'effetto di rendere più difficile la coesione e quindi l'istituzionalizzazione ${ }^{4}$ (tab. 4). Infatti, un reclutamento che scavalca le articolazioni locali riduce la lealtà verso la subunità organizzativa in cui l'eletto è inserito, a vantaggio di quella verso il centro patrimoniale del partito nel caso di un reclutamento effettuato da parte di dirigenti nazionali, o verso constituencies extra-partitiche, o addirittura extra-politiche nel caso di reclutamento tramite club o amici. Per questo secondo gruppo di eletti si pone dunque un problema di accountability nei confronti della gerarchia locale del partito.

Un ulteriore fattore di dipendenza-indipendenza dell'eletto dai selectorates, e dunque di capacità di controllo da parte del partito e di coesione organizzativa, è legato alla direzione del reclutamento. La dipendenza dell'eletto sarà maggiore se egli è stato sollecitato a candidarsi dal partito stesso, che lo ha individuato e mobilitato attivamente (reclutamento attivo) offrendogli la possibilità di essere eletto attraverso la persona del selezionatore. Se invece è stato l'eletto ad avanzare la propria candidatura (reclutamento passivo), egli godrà di una maggiore indipendenza, in quanto si è mostrato «necessario» al partito. Sarà quindi meno inquadrabile e la sua lealtà più difficile da incanalare. Nel caso di Forza Italia, l'offerta di candidature locali da parte del partito è stata superiore alla domanda. Di conseguen$\mathrm{za}$, il partito è stato in molti casi indotto ad accettare l'autocan-

4 Quasi un quarto dei contatti è passato attraverso il canale più informale degli amici attivi nel movimento e un quinto attraverso i canali extra-gerarchici dei club e della dirigenza nazionale del partito. Questa modalità di reclutamento, alternativa a quella verticale-gerarchica, interessa globalmente quasi un terzo del campione, e contribuisce a precostituire una situazione di indeterminatezza dei ruoli e di incertezza nella distribuzione degli incentivi organizzativi. 
TAB. 5. Modalità di reclutamento: da chi è stato contattato/chi ha contattato (\%)

\begin{tabular}{lcc}
\hline Modalità del contatto & È stato contattato da & Ha contattato \\
\hline Esponenti di FI: & & \\
Coordinatore regionale & 14,7 & 16,0 \\
Coordinatore provinciale & 12,0 & 4,0 \\
Delegato di collegio & 9,3 & 16,0 \\
Parlamentare & 12,0 & 6,0 \\
Europarlamentare & 1,3 & 2,0 \\
Amici attivi in FI & 14,7 & 40,0 \\
Presidente di club & 10,9 & 2,0 \\
Dirigente nazionale & 17,1 & 6,0 \\
Più contatti & 8,0 & 8,0 \\
Totale & 100,0 & 100,0 \\
\end{tabular}

didatura dei potenziali aspiranti, semplicemente per la necessità di riempire le liste. Nel campione in esame abbiamo rilevato un $60 \%$ di casi di reclutamento attivo e un $40 \%$ di autocandidature. La disaggregazione di questo dato globale, che si riferisce indifferentemente al soggetto attivo o passivo del reclutamento, evidenzia la predominanza di soggetti di reclutamento diversi al variare della direzione del contatto (tab. 5). Il reclutamento attivo ha avuto luogo prevalentemente tramite la dirigenza locale. Tuttavia, il reclutamento da parte di reti più informali, societarie in senso lato, ha interessato un quarto degli eletti reclutati attivamente. Sembrerebbe trovare conferma, limitatamente a questo gruppo di eletti, l'ipotesi di un modello centralizzato e controllato dalla dirigenza locale di Forza Italia: infatti, il 48\% di coloro che sono stati contattati lo è stato da parte del coordinatore regionale, del coordinatore provinciale, e del delegato di collegios.

Inoltre, anche l'élite nazionale con i parlamentari da un lato $(13,3 \%)$, e altri membri della dirigenza dall'altro $(17,1 \%)$ si è data da fare attivamente per trovare candidati. Complessivamente, gli eletti reclutati attraverso il canale partitico sono il $78,4 \%$. Vi è tuttavia una parte consistente di eletti che è stata contattata attraverso canali più informali, come gli «amici attivi

5 Si tratta di dirigenti locali nominati tutti dal vertice fino alla fine del 1997, quando ex statuto il coordinatore provinciale viene eletto a scrutinio segreto dal congresso provinciale. Il coordinatore regionale, che nomina i delegati di collegio, continua a essere nominato dal presidente. Fino alla riforma del 1997 questi ruoli sono stati tutti ricoperti, ove possibile, da personale di matrice aziendale (Poli 1997). 
nel movimento» $(14,7 \%)$ e i presidenti dei club Forza Italia $(10,9 \%)$. Queste modalità di contatto delineano un diverso percorso di accesso extra-gerarchico all'arena politica, che ha interessato un quarto degli eletti reclutati attivamente (pari al $12,5 \%$ del totale del campione).

Diversa è la struttura del contatto nel reclutamento che prende la direzione opposta. Le autocandidature di coloro che si sono presentati al partito di propria iniziativa, offrendosi come potenziali candidati, si sono indirizzate soprattutto a soggetti con i quali l'aspirante intratteneva rapporti amicali o comunque informali. Si abbassa la percentuale di coloro che sono transitati attraverso il canale dei dirigenti partitici locali, l'attivazione presso il parlamentare è molto più esigua che nel reclutamento attivo, ma è soprattutto il canale extra-gerarchico che tende a dilatarsi smisuratamente. Infatti, pur restando il reclutamento attraverso il canale partitico quello prevalente, ben il $40 \%$ degli eletti ha contattato «amici attivi nel movimento». Si tratta di una importante categoria di eletti, che probabilmente sono meno inquadrabili e controllabili da parte dei dirigenti locali, poiché sfuggono al legame diretto di lealtà patrimoniale, e dunque si trovano più lontani da quel centro del partito da cui promana la legittimità.

Globalmente, ben un quarto degli eletti è stato reclutato con modalità amicale extra-partitica ed extra-gerarchica, per non dire extrapolitica tout court, ed ha di conseguenza un aggancio labile con l'apparato personale del leader 6 . Se tuttavia consideriamo tutti gli autocandidati come potenzialmente extragerarchici, allora abbiamo in più della metà dei casi un reclutamento produttivo di una forma di lealtà extra-partitica. Se infatti si realizza anche in questi casi quella modalità di rapporto tra selectorate e eletto che Putnam individua come fondamentale aspetto del processo di reclutamento, ovvero l'indirizzarsi della lealtà del selezionato verso il gruppo che lo ba prescelto, ebbene, la lealtà di questi eletti non si indirizzerà direttamente al partito, ma sarà mediata da altre strutture più fluide e poco controllabili, come le reti amicali. Questo risultato rivela una ambiguità di fondo a partire dalla prima fase di contatto tra

6 Questa percentuale è ottenuta sommando il $12,5 \%$ del campione che è stato reclutato attivamente da amici con il $13,5 \%$ di chi si è autocandidato presso amici attivi nel movimento. 
TAB. 6. Eletti locali di FI con precedenti esperienze politiche (cariche partitiche elo amministrative in enti locali)

\begin{tabular}{lcc}
\hline Eletti & N & $\%$ \\
\hline Senza precedenti esperienze politiche & 91 & 56,9 \\
Con precedenti esperienze politiche & 69 & 43,1 \\
Totale & 160 & 100,0 \\
\hline
\end{tabular}

Forza Italia e quello che poi diventerà il suo personale politico locale, che prelude ad una difficoltà di istituzionalizzazione dovuta a una scarsa coerenza organizzativa.

Il ruolo dei selezionatori è importante anche perché essi definiscono le caratteristiche necessarie per essere prescelti. Le caratteristiche qualitative dei selezionati su cui ci concentreremo in questo articolo sono relative alla presenza o meno di una precedente esperienza politica (partitica o amministrativa) e alla professione dell'eletto. L'ipotesi di partenza tiene conto della politica visibile (Pizzorno 1993, 286) e del messaggio ideologico di Forza Italia, prevedendo che nel reclutamento attivo del partito prevalgano da un lato i candidati nuovi, ovvero estranei alla vecchia politica, e dall'altro le professioni congruenti, ovvero quelle che il discorso pubblico del partito, che ne consente una identificabilità e distinguibilità palese, esalta come portatrici dei valori che esso intende promuovere: medi e piccoli imprenditori, manager, liberi professionisti, artigiani ed esercenti.

Per quanto riguarda le precedenti esperienze politiche, abbiamo controllato l'appartenenza degli eletti alla categoria del professionismo politico, creata sommando coloro che, prima di entrare in Forza Italia, hanno ricoperto cariche direttive in altri partiti, oppure cariche amministrative a livello locale. Le risposte ottenute consentono di collocare in questa categoria il $43,1 \%$ degli intervistati (tab. 6).

Dall'incrocio di questo dato con la direzione seguita dal reclutamento emerge che il personale con precedenti esperienze politiche è stato reclutato attivamente dal partito in misura soltanto lievemente inferiore al personale che ne era privo. Viceversa, la possibilità di autocandidarsi è stata sfruttata assai poco dagli ex politici di professione, e invece in misura massiccia da parte del personale nuovo alla politica, gli amateurs. Infatti, tra i reclutati attivamente più della metà sono ex politici locali, mentre tra gli eletti che si sono spontaneamente attivati gli ex 
TAB. 7. Modalità di reclutamento degli eletti con precedenti esperienze politiche (\% di riga e di colonna)

\begin{tabular}{lcccc}
\hline Eletto & $\begin{array}{c}\text { Senza esperienza } \\
\text { precedente }\end{array}$ & \multicolumn{2}{c}{$\begin{array}{c}\text { Con esperienza } \\
\text { precedente }\end{array}$} \\
\hline È stato contattato & 54 & 56 & 47 & 67 \\
Ha contattato & 65 & 44 & 35 & 33 \\
\hline
\end{tabular}

politici sono solo poco più di un terzo. Del totale degli ex professionisti della politica presenti tra gli intervistati dunque $2 / 3$ sono stati contattati dal partito, e solo $1 / 3$ si è attivato spontaneamente (tab. 7).

Alla dicotomia nel modello di reclutamento, che vede affiancarsi a un modello centripeto d'apparato e prevalere su di esso un sistema più aperto, ma centrifugo, si somma una ulteriore dicotomia tra coloro che, contattati da Forza Italia, risultano essere in quasi la metà dei casi ex professionisti della politica $^{7}$, e un personale amatoriale che si attiva per $2 / 3$ di propria iniziativa. Il dato più interessante emerge però dall'incrocio di quest'ultima categoria con il soggetto del contatto. Ebbene, questi eletti dilettanti si sono attivati per il tramite extra-politico degli amici attivi nel movimento in misura tre volte superiore ai politici locali di professione. Vi è dunque una presenza rilevante di reclutamento societario che passa attraverso l'autocandidatura tramite amici o presidenti di club, e che prelude a una bassa coerenza organizzativa. Il reclutamento attivo di ex professionisti della politica denota invece una scarsa autonomia dall'ambiente e dunque chances ridotte di istituzionalizzazione. Questi eletti infatti hanno un atteggiamento ambivalente nei confronti del partito. Essi hanno percepito la propria posizione come incongrua rispetto allo stile e ai valori di Forza Italia e, anticipando la possibilità di un fallimento della propria candidatura, non si sono attivati di propria iniziativa che in numero relativamente limitato (Norris e Lovenduski 1997, 162). Il partito invece, contrariamente a tali aspettative «razionali» e alla

7 Per quanto riguarda la provenienza partitica degli eletti già aderenti in passato ad altre formazioni politiche $(56,2 \%)$ il $45,6 \%$ era iscritto alla Dc e il $22,2 \%$ al Psi. Complessivamente, i provenienti dall'area dell'ex pentapartito sono 1 ' $88,9 \%$ dei già iscritti ad altro/i partito/i. Sul background degli eletti si veda Tonarelli (1999). 
propria linea ufficiale ${ }^{8}$, ha attinto a piene mani da questo gruppo, con la conseguenza che le sue fila si sono ingrossate di ex professionisti provenienti dalle retrovie della politica locale, reclutati prevalentemente attraverso la struttura gerarchica del partito, che riescono in tal modo a riprendere le modeste carriere prematuramente interrotte ${ }^{9}$. L'ipotesi della congruenza tra le caratteristiche richieste dai selezionatori e il messaggio pubblico di Forza Italia viene pertanto smentita. Si evidenzia invece un forte scostamento della politica occulta da quella visibile. Che cosa può avere indotto i selezionatori di Forza Italia a ricorrere in misura così massiccia ai politici della Prima repubbli$c a$ ? Essi hanno probabilmente agito in presenza di due imperativi forti: la scarsità di candidature e l'opportunità di ricorrere a personale già esperto e inserito nella politica locale. La politica occulta, infatti, a differenza di quella palese e pubblica, non prescinde mai dagli interessi materiali dei soggetti, anzi è volta precisamente alla soddisfazione di fini che devono restare invisibili (Pizzorno 1993, 287). La lealtà e la continuità della partecipazione di questi eletti dipenderà in larga misura dalla capacità di Forza Italia di distribuire loro sufficienti incentivi selettivi. Se infatti si ipotizza che gli ex politici del pentapartito siano dei carrieristi (Panebianco 1982), ebbene, essi vorranno che il partito favorisca la propria carriera nelle istituzioni (fornendo sostegno in campagna elettorale e appoggio nello svolgimento dei propri compiti istituzionali) e renda possibile una carriera interna (attuando una chiara definizione della mappa del potere organizzativo, ed elaborando percorsi di carriera strutturati). La loro partecipazione è comunque sempre in forse, e dipende direttamente dalla capacità del partito di fornire gli incentivi richiesti. Gli amateurs, invece, mobilitatisi in larga misura autonomamente, essendo consci della propria funzione ideologica di portabandiera delle nuove classi medie, saranno più sensibili

$8 \mathrm{La}$ linea ufficiale, ribadita da Berlusconi in occasione del Congresso del 1998, è la seguente: «Forza Italia è la vera novità della politica italiana. Rispetto e in antitesi agli altri movimenti politici FI non ha atti, scelte, presenze, responsabilità, radici affondate nella Prima Repubblica. Questa la sua novità e la sua forza. Forza Italia non appartiene al passato ma nasce dalla grande speranza e dalla grande voglia di cambiamento della gente, dall'esigenza di profondo rinnovamento del sistema e del costume politico italiano». Cfr. Berlusconi (1998).

9 La modestia delle carriere può essere dedotta dalla bassa percentuale di coloro che, avendo ricoperto cariche politico-amministrative in passato $(34,4 \%)$ dichiarano $\mathrm{di}$ aver avuto incarichi di rilievo (26,9\%). Cfr. Tonarelli (1999). 
alla realizzazione dei fini organizzativi ufficiali, e dunque saranno più attenti alla capacità del partito di produrre politiche corrispondenti al messaggio ufficiale. Essi dovranno essere premiati con incentivi collettivi e di status, più che con incentivi di potere, carriera o materiali. Vedremo più avanti se queste condizioni sono state soddisfatte dal partito.

Oltre che come manifestazione del nuovo, Forza Italia ha ritratto i propri eletti come espressione diretta del ceto medio produttivo, che quindi sarebbe rappresentato di fatto senza il filtro di professionisti della politica (Verzichelli 1997b). Si è ritenuto di accertare se tale corrispondenza tra appartenenza di ceto e carica rappresentativa sia stata effettivamente realizzata, almeno nelle intenzioni dei selezionatori. Tale impegno sarebbe stato la prova della capacità e volontà di mobilitazione di determinati gruppi socio-economici, e quindi della compattezza e coerenza potenziale del partito, nonché degli eletti in quanto gruppo (Gallagher e Marsh 1988, 15).

Abbiamo suddiviso le professioni dei nostri intervistati in professioni congruenti o incongruenti con il messaggio politicoculturale e le linee programmatiche di Forza Italia ${ }^{10}$. Tra le prime rientrano molte delle professioni tipiche dei professionisti della politica della Prima repubblica (politici di professione, professori universitari e magistrati, tecnici, impiegati del settore pubblico, coltivatori diretti, studenti, politici e sindacalisti), delle seconde fanno parte invece artigiani e commercianti, imprenditori, liberi professionisti e dirigenti d'azienda. Come si evince dalla tabella 8 , il reclutamento attivo si è rivolto a tutti i gruppi professionali rappresentati nel nostro campione, anche se con notevoli variazioni di intensità. Il partito recluta un numero maggiore di eletti con professioni congruenti, ma un quarto dei reclutati svolge professioni non congruenti. Nel reclutamento passivo, al contrario, figurano quasi esclusivamente professioni congruenti.

Anche il tipo di professione congruente varia a seconda del modello di reclutamento. Attivamente Forza Italia recluta alcuni imprenditori, ma soprattutto liberi professionisti e manager, mentre il reclutamento passivo vede una maggiore attivazione di artigiani e imprenditori. Pertanto, a differenza di ciò che emerge dalle dichiarazioni ufficiali a livello della politica visibi-

10 Si ringrazia Alberto Marradi per aver messo a disposizione di chi scrive la sua classificazione delle professioni, che ha facilitato il corretto trattamento ex post delle risposte alla domanda aperta relativa alla professione dell'eletto. 
TAB. 8. Modello di reclutamento: reclutamento passivo/attivo e professione congruente I (valori assoluti e percentuale del totale del campione)

\begin{tabular}{lccc}
\hline & $\begin{array}{c}\text { Reclutamento } \\
\text { attivo }\end{array}$ & $\begin{array}{c}\text { Reclutamento } \\
\text { passivo }\end{array}$ & Totale \\
\hline $\begin{array}{l}\text { Professioni non congruenti } \\
\text { Professioni congruenti }\end{array}$ & $30(22,0)$ & $18(13,2)$ & $48(35,2)$ \\
\hline
\end{tabular}

ТАВ. 9. Modello di reclutamento: reclutamento passivo/attivo e professione congruente II $(\%)$

\begin{tabular}{lcc}
\hline Modalità del contatto & Reclutamento attivo & Reclutamento passivo \\
\hline Professione del reclutato: & & \\
Commercianti e artigiani & 16,7 & 83,3 \\
Imprenditori & 46,7 & 53,3 \\
Liberi professionisti & 66,0 & 34,0 \\
Dirigenti d'azienda & 64,3 & 35,7 \\
\hline
\end{tabular}

le, i selectorates di Forza Italia per le elezioni amministrative non disdegnano, e sarebbe difficile farlo data la penuria di aspiranti, i candidati dalla professione non congruente, e anche quando reclutano all'interno delle professioni congruenti attingono in prevalenza ad alcuni particolari segmenti del ceto medio, soprattutto i liberi professionisti, gli avvocati, i commercialisti e $\mathrm{i}$ dirigenti d'azienda che, a differenza di artigiani, commercianti, ma anche imprenditori, si attivano molto meno di propria iniziativa (tab. 9).

Sul versante della professione degli eletti emergono pertanto ulteriori ambiguità nel modello di reclutamento, e quindi nella offerta di rappresentanza. Vi è certo da parte di Forza Italia la volontà di far coincidere rappresentanza politica e rappresentatività sociologica degli eletti ${ }^{11}$. Questo nuovo soggetto dell'arena competitiva italiana si è fatto portatore di una visione anti-partitica se non anti-politica ed economicistica della rappresentanza favorendo l'infusione diretta nella sfera politica di valori e capacità propri di quel mondo che essa privilegia a livello ideologico, ovvero quello dell'impresa, della libera professione e del lavoro autonomo. La pretesa negatività della mediazione partitica

11 Si tratta per altro di una tendenza generale dei partiti italiani, come rilevato da Bartolini e D'Alimonte (1997, 358). 
porta inevitabilmente a cercare di far coincidere la propria offerta di rappresentanza con i ceti sociali privilegiati a livello programmatico. Tuttavia, la certo massiccia presenza di figure professionali che rappresentano i settori dell'economia privata, come gli imprenditori e i dirigenti d'azienda è stata solo parzialmente il frutto intenzionale di un reclutamento mirato ad incorporare negli eletti $i$ valori più evidenti e unificanti per l'intero movimento. Se è pur vero che la composizione sociale della maggioranza del campione indica l'attribuzione di un netto privilegio al settore economico privato, è anche vero che la autocandidatura di questi ceti è stata superiore alla loro mobilitazione da parte di Forza Italia, la quale ha reclutato intensamente solo i liberi professionisti (è la categoria «congruente» con il più alto scarto tra reclutamento attivo e passivo, ben 32 punti) $\mathrm{e}$ in subordine $\mathrm{i}$ dirigenti d'azienda (anche qui lo scarto è notevole - 28,6 punti - ma la cifra assoluta è molto più bassa). Queste due categorie, ma soprattutto la prima, rappresentano quei settori della nuova classe media, o classe di servizio, che svolgono principalmente una funzione di supporto agli interessi piuttosto che di rappresentanza degli stessi ${ }^{12}$. Queste professioni sembrerebbero dunque prendere in Forza Italia il posto occupato negli altri partiti della Prima repubblica dal funzionariato pubblico-partitico (Tonarelli 1999).

Tuttavia, sebbene si cerchi di reclutare maggiormente tra questi settori nuovi alla politica attiva, non si disdegnano i vecchi serbatoi della classe politica, che hanno il vantaggio di fornire legami, clientele e voti potenzialmente disponibili in quanto rimasti privi di referenti politici. Questo atteggiamento pragmatico è probabilmente determinato sia dalla carenza di personale candidabile, sia dal bisogno di competenze politiche locali, che induce $\mathrm{i}$ dirigenti ad allargare la selezione alle professioni che hanno tradizionalmente fornito la maggior quota di eletti nei partiti italiani (Verzichelli 1997a, 341). E tuttavia significativo che le professioni non congruenti con il messaggio politico neo-liberista e anti-statalista di Forza Italia si attivino molto meno di quanto non siano invece sollecitate a farlo da parte di Forza Italia. Come anche nel caso degli ex politici locali, vi è da parte di queste professioni un certo «sospetto» nei confronti di

12 «L'esercizio di autorità delegata è [il servizio] tipico dei dirigenti (manager) [...]. La conoscenza e la competenza sono invece caratteristiche tipiche dei servizi offerti dai liberi professionisti» (Kriesi 1998, 59). 
Forza Italia, derivante dalla percezione del suo messaggio politico come antagonistico, se non ostile. Pur non trattandosi di gruppi molto disponibili alla mobilitazione spontanea in Forza Italia, né sensibili al suo richiamo ideologico, tra di essi vengono reclutati attivamente dal partito quasi un quinto degli eletti. Se è dunque vero che il background socio-professionale della maggioranza degli intervistati si conforma con la cultura politica e il programma di Forza Italia, sembra tuttavia che la minore presenza delle categorie non congruenti non sia tanto il frutto di una deliberata esclusione, quanto di una mancata attivazione spontanea, di una ritrosia nei confronti dell'offerta di candidature, che pure c'è stata, e abbastanza consistente, da parte di Forza Italia. I magistrati, i sindacalisti, i coltivatori diretti, i giornalisti e gli intellettuali presenti tra gli eletti di Forza Italia sono infatti stati tutti reclutati attivamente dal partito. Solo gli insegnanti e i funzionari e dipendenti pubblici si sono attivati a loro volta in numero elevato, almeno pari a quello dei reclutati dal partito. Ma si tratta in questi casi di figure tradizionalmente dedite alla politica, nonché di professioni a larghissima diffusione, dotate di risorse, soprattutto di tempo, alle quali è difficile che la politica possa rinunciare da un giorno all'altro. Queste professioni sono portate allo sviluppo di lealtà organizzative, ma per garantirne la partecipazione il partito dovrà offrire loro incentivi di solidarietà. Viceversa, l'alto numero di liberi professionisti reclutati può porre seri problemi di persistenza organizzativa. Infatti, a differenza del funzionariato classico, essi non sono affatto privi di opzioni di exit. Al contrario, per loro il grado di sostituibilità degli incentivi è molto elevato. Se il partito non è in grado di fornire sufficienti incentivi di carriera, visibilità e status vi è un'alta probabilità di uscita. Pertanto, non si tratta certo di un personale che favorisce l'istituzionalizzazione del partito ma che, al contrario, a seconda delle opportunità tenderà indifferentemente ad entrare o uscire attraverso la porta girevole della politica (Norris 1997, 4).

Più che un partito che consapevolmente e autonomamente si fa garante della copertura politica delle istanze dei ceti medi imprenditoriali, tutelati fino a pochi anni fa dalla Dc, siamo di fronte a una forza politica che quasi sembra subire l'entusiasmo di questi ceti, che in essa si riversano fiduciosi mostrando di identificarsi con il suo messaggio programmatico. Lo mostra l'attivazione spontanea di artigiani ed esercenti, che sono reclutati invece in misura minima dal partito. Dunque Forza Italia si 
presenta più come il passivo recettore della partecipazione di alcuni gruppi sociali, che essa ha peraltro sollecitato in misura decisiva, piuttosto che come il soggetto attivo di un riallineamento socio-politico (Dalton et al. 1984). Questa interpretazione viene confermata dall'analisi delle modalità di organizzazione e conduzione della campagna elettorale dei candidati alle elezioni amministrative.

\section{La campagna elettorale}

Durante la campagna elettorale di norma inizia a prendere corpo lo scambio tra incentivi e partecipazione, e si pongono le basi per un rapporto più o meno stretto e soddisfacente tra il partito e il futuro eletto. Condividere con il candidato l'impegno della campagna elettorale fornisce all'eletto una forte motivazione alla partecipazione e all'identificazione con il partito ${ }^{13}$.

Dalla batteria di domande del nostro sondaggio dedicate alla campagna elettorale dell'eletto si possono pertanto trarre indicazioni sulla volontà e capacità di radicamento territoriale del partito e sul grado di sviluppo della lealtà organizzativa a livello locale.

Il ruolo svolto dal partito nel sostegno delle campagne elettorali locali è un buon indicatore di quanto Forza Italia sia interessata a svilupparsi a livello periferico - se infatti lo è, deve anche essere disposta a organizzarsi in maniera più capillare, e a dare un certo supporto logistico, organizzativo ed anche finanziario ai propri candidati. L'aspetto finanziario in particolare può fornire una chiarificazione in merito al rapporto tra centro e periferia: a fronte dell'enorme dispendio di energie e mezzi profusi nella propaganda a livello nazionale, il partito che atteggiamento adotta in caso di elezioni locali, ovvero quanto decide di investire nelle campagne dei propri candidati?

Le risposte degli eletti relative al contributo finanziario ricevuto dal partito o da altri sostenitori alla propria campagna, sintetizzate nella tabella 10, attestano il manifesto disinteresse da parte di Forza Italia verso un impegno diretto a sostegno dei

13 Con riferimento ad un gruppo di candidati repubblicani statunitensi, tra gli incentivi di solidarietà Roback inserisce anche la condivisione dell'impegno della campagna elettorale, che contribuisce ad una forte motivazione alla partecipazione iniziale del candidato (Roback 1974, 21). 
TAB. 10. Finanziamento della campagna elettorale (\%)

\begin{tabular}{lccc}
\hline $\begin{array}{l}\text { Tipo di } \\
\text { finanziamento }\end{array}$ & $\begin{array}{c}\text { Nessun } \\
\text { finanziamento }\end{array}$ & $\begin{array}{c}\text { Finanziamento parziale } \\
(\text { dal 10 al 90\%) }\end{array}$ & $\begin{array}{c}\text { Finanziamento } \\
\text { totale }\end{array}$ \\
\hline Partito & 93,6 & 5,8 & 0,6 \\
Mezzi propri & 3,8 & 36,9 & 58,3 \\
Sostenitori & 62,7 & 35,4 & 1,9 \\
\hline
\end{tabular}

candidati: il 94\% degli intervistati dichiara di non aver ricevuto alcun contributo finanziario dal partito, mentre un unico candidato ha goduto di una copertura totale dei costi della propria campagna.

Ma le risposte alla domanda sul finanziamento contengono altri elementi d'interesse. Più della metà degli eletti ha coperto l'intera spesa della campagna elettorale con fondi propri ${ }^{14}$. A differenza di quanto ci si sarebbe potuti aspettare in un partito che si autorappresenta come organizzato sulla falsariga del modello americano e che in più candida un alto numero di persone in quanto rappresentanti di specifiche categorie economiche, i contributi dei sostenitori sono assai scarsi. Ben il $63 \%$ degli eletti non ha ricevuto alcun contributo da parte di sostenitori. Oltre a confermare la distanza rispetto al modello americano del sistema di competizione maggioritaria all'italiana (Bartolini e D'Alimonte 1997), ciò mette in evidenza anche un fenomeno molto eloquente, ovvero che le associazioni di categoria non appoggiano i propri rappresentanti. Ciò è tanto più evidente, quanto più gli eletti di Forza Italia non si limitano a rappresentare «virtualmente» gli interessi delle categorie, ma spesso appartengono ad associazioni di categoria in qualità di iscritti o dirigenti ${ }^{15}$.

Da queste prime indicazioni sull'appoggio finanziario emerge sia un quadro di abbandono dei candidati da parte del parti-

14 Si tratta di persone che hanno una certa disponibilità finanziaria, il che è più che plausibile, trattandosi in buona parte di appartenenti a strati medi o medio alti, con un'istruzione di livello universitario. Il $66 \%$ degli intervistati è laureato, mentre solo il $3,6 \%$ non è andato oltre la scuola dell'obbligo. Sulle caratteristiche demografiche del campione si veda Tonarelli (1999).

15 Il $44,4 \%$ del campione ha fatto o fa parte di associazioni, il $28,1 \%$ vi ha ricoperto o vi ricopre cariche direttive. Gli appartenenti a associazioni di rappresentanza d'interessi economici sono il 35,4\%. Cfr. Tonarelli (1999). 
TAB. 11. Appoggio ricevuto in campagna elettorale (\%)

\begin{tabular}{lcc}
\hline & Nullo/scarso & Forte \\
\hline Coalizione & 61,9 & 6,9 \\
Partito & 42,5 & 20,0 \\
Comitato elettorale & 33,8 & 37,5 \\
Amici & 6,3 & 75,0 \\
Categorie professionali/sindacati & 48,1 & 16,9 \\
Associazioni cattoliche & 63,1 & 7,5 \\
Club FI & 46,3 & 17,5 \\
Stampa locale & 66,9 & 5,0 \\
Coordinatore regionale FI & 57,5 & 15,0 \\
\hline
\end{tabular}

to, sia, contrariamente alla retorica della mobilitazione della società civile, una scarsa attivazione da parte di quest'ultima, che tende invece a sottrarsi all'impegno diretto o quanto meno collaterale nella battaglia politica. Nell'attuale fase di crisi e cambiamento dei referenti politici tradizionali, il mondo associativo non sembra volersi inserire nella contesa politica, quanto meno a livello locale (Ferrante 1998, 113).

Il disinteresse di Forza Italia che si manifesta nella quasi totale assenza di incentivi materiali sotto forma di contributi finanziari alla campagna elettorale viene ribadito dall'assenza di qualsiasi sostegno più generale, come un appoggio logistico o psicologico, un sostegno quest'ultimo certo più immateriale, ma comunque importante ai fini della creazione di un legame organizzativo stabile, in quanto implica la distribuzione di incentivi collettivi atti a suscitare il senso di appartenenza (Pozzi e Rattazzi 1994). Quasi la metà degli eletti ritiene di non aver ricevuto alcun appoggio alla propria campagna elettorale da parte di Forza Italia (tab. 11). Solo per $1 / 5$ di essi l'appoggio del partito è stato forte, mentre per $1 / 3$ è stato soltanto discreto. Nella percezione dei propri eletti Forza Italia dunque si mostra scarsamente interessata al sostegno dei candidati locali, dando un segnale di bassa integrazione organizzativa.

Se il sostegno da parte del partito considerato come entità collettiva è stato molto basso, abbiamo ipotizzato l'esistenza di un appoggio maggiore da parte di eventuali correnti interne in via di formazione o subunità del partito, ma ne abbiamo subito riscontrato l'assenza. Infatti sia i club sia i coordinatori regionali posti a capo della organizzazione locale danno un sostegno scarso o nullo alla maggioranza dei candidati. L'appoggio ricevuto dai club di Forza Italia è stato ritenuto insufficiente da 
quasi la metà degli eletti, ed è stato considerato soltanto discreto da un terzo di essi. Si conferma così il ruolo ormai secondario dei club, dovuto a una marginalizzazione legata sia alla mancanza di risorse sia ad una collocazione organizzativa non integrata nella struttura del partito ${ }^{16}$. Essi non sono in grado pertanto di fungere da eventuale massa di manovra per subleader intenzionati ad organizzare proprie autonome fazioni. D'altra parte, anche l'appoggio del coordinatore regionale di Forza Italia risulta insufficiente per addirittura il 60\% degli eletti, ed è considerato forte solo da uno scarno $15 \%$ di essi. Queste cifre gettano luce sullo stato dei rapporti tra centro e periferia del partito. Essendo il plenipotenziario del centro sul territorio il coordinatore regionale dovrebbe svolgere un'azione di raccordo e di armonizzazione. In realtà, questa figura sembra avere una funzione di barriera tra la base e i vertici del partito. L'integrazione verticale ne risulta pertanto assai indebolita, e la formazione di fazioni inibita da tale cesura tra dirigenza nazionale e livelli subregionali.

Visto che dal partito non è arrivato alcun appoggio forte alla carriera politica dei candidati, che sembrano interamente abbandonati a se stessi e impossibilitati, per motivi strutturali, ad organizzarsi in subunità che in qualche modo ne favorirebbero l'identificazione organizzativa, abbiamo ipotizzato la presenza di un maggiore appoggio da parte di simpatizzanti e categorie economiche, sulle quali la propaganda politica di Forza Italia ha insistito molto. Ebbene, anche la società civile organizzata sembra aver adottato un atteggiamento di attendismo e prudenza: se il suo sostegno finanziario è parso quasi inesistente, è mancato anche un appoggio più generico. Nella metà dei casi quest'ultimo è stato addirittura nullo, a conferma della scarsa mobilitazione delle categorie e della società civile organizzata. Solo nel $17 \%$ dei casi il loro appoggio in campagna elettorale è stato forte (tab. 11). La debolissima mobilitazione

16 Lo statuto del 1997 formalizza il disinteresse mostrato nel corso degli anni precedenti dalla leadership nei confronti dei club, e la sua volontà di non dare vita a strutture che possano assomigliare alle sezioni locali dei partiti tradizionali. La nona parte dello statuto, dedicata ai «Rapporti con i club Forza Italia», li denota come strutture esterne, pertanto ininfluenti nel processo decisionale del partito, eppure tenute ad accettarne la linea politica, e assoggettate al coordinamento delle proprie iniziative aventi valenza politica da parte dei dirigenti locali di Forza Italia. Tale rapporto di scambio fortemente ineguale può funzionare solo in condizioni particolari, ma alla lunga l'assenza di incentivi e il presentarsi di alternative tenderanno a far prevalere l'opzione di uscita. 
delle associazioni e delle categorie risulta non soltanto confermata, ma addirittura significativamente accentuata nel caso delle associazioni cattoliche. Nel $63 \%$ dei casi il loro appoggio è stato nullo o scarso. La mobilitazione della società civile cattolica risulta di 15 punti inferiore rispetto a quella, pur bassa, delle categorie economiche. Si tratta di conferme della mancata ricostituzione a livello locale dei rapporti tradizionali di delega tra partito e associazioni collaterali. Le organizzazioni non si impegnano per far eleggere i propri rappresentanti nelle amministrazioni locali tramite il canale di Forza Italia, pur avendo questa assunto su di sé programmaticamente il compito di rappresentare le nuove classi medie, che molte associazioni tutelano. Probabilmente il declino della polarizzazione ideologica, la mancanza di identificazione tra gruppi dirigenti associativi e dirigenza di Forza Italia (i cui i ruoli chiave come quello del coordinatore regionale sono ricoperti in maggioranza da personale di provenienza diretta o indiretta Fininvest), la debole integrazione del partito, l'assenza di procedure che favoriscano l'identificazione collettiva dei partecipanti con l'organizzazione, la sua scarsa articolazione territoriale hanno inciso in maniera negativa sull'attivazione diretta delle categorie a sostegno dei candidati (Ferrante 1998, 98). Del resto, in questa fase di transizione sistemica prevale un cauto attendismo e un atteggiamento di neutralità dei gruppi rispetto ai partiti (Morlino 1991).

Lo scollamento dell'eletto dal partito e dalla società civile organizzata si riflette nella percezione dell'atteggiamento della stampa locale. Gli eletti percepiscono di essere stati penalizzati dai mezzi di informazione. Il sostegno, quanto meno informativo, di questi ultimi alla campagna elettorale è ritenuto insufficiente dal $67 \%$, e forte solo dal 5\% degli intervistati. Anche questo è un dato molto significativo rispetto alla capacità di mobilitazione a livello locale da parte di Forza Italia, in quanto esprime la percezione diffusa di una condizione di inferiorità e impotenza rispetto ai c.d. poteri forti della società, tra cui la stampa e gli altri media locali, che si evidenzierà anche nella risposta alla domanda successiva sui mezzi utilizzati per la campagna, e che affonda le sue radici in un problema di mancata integrazione dell'organizzazione nel sistema sociale locale. L'inefficacia organizzativa del partito in campagna elettorale si manifesta altresì nella percezione negativa del sostegno ricevuto dalla coalizione del Polo, che riflette una ulteriore debolezza, di tipo negoziale, della dirigenza locale del partito. 
Constatato l'atteggiamento poco efficace o distaccato del partito e degli altri attori sociali rilevanti, il candidato ha dovuto crearsi una propria struttura ad boc, che ne organizzasse e sostenesse la campagna, il comitato elettorale. Quasi la metà degli eletti dichiara di essere stato sostenuto in modo forte da questo tipo di struttura personale. Tuttavia, un quarto non lo ha utilizzato, e un altro quarto ne è rimasto insoddisfatto. Non può non aver influito sull'alta percentuale di insoddisfatti o di coloro che non lo hanno utilizzato la scarsità di finanziamenti che i candidati sono riusciti a ottenere sia dal partito che dalle categorie e dai sostenitori ${ }^{17}$. Infine, $3 / 4$ degli intervistati dichiarano di aver avuto un appoggio forte da parte degli amici. La rilevanza del comitato elettorale extra-partitico privato e degli amici nella gestione della campagna possono essere letti come indicatori della dimensione non ideologica, privatistica della partecipazione politica in Forza Italia, ma anche di un rapporto di tipo franchising tra candidati e partito, in base al quale il partito mette a disposizione il proprio marchio, che viene gestito dal candidato in piena autonomia e con l'aiuto della propria cerchia amicale e di strutture ad hoc autofinanziate, anziché dall'organizzazione partitica. La maggiore autonomia del candidato, associata troppo spesso a dilettantismo ed inefficienza, contribuisce a mantenere basso il livello di coesione dell'organizzazione.

Una conferma della privatizzazione del rapporto tra eletto e partito e della scarsa coesione interna viene dall'analisi delle risposte alla domanda sugli strumenti di propaganda politica utilizzati (tab. 12). Contrariamente alle campagne nazionali di Forza Italia, a livello periferico il ricorso allo spot televisivo è stato precluso all' $86 \%$ dei candidati. Il $92 \%$ degli eletti non ha nemmeno fatto ricorso a spot radiofonici. Le inserzioni sui giornali possono essere assimilate allo spot, anche se di tipo più tradizionale. Se ne è servito solo poco più di un quarto degli intervistati. Questi dati sono coerenti con il basso profilo finanziario già emerso. $\mathrm{Ma}$ anche la partecipazione a trasmissioni radiotelevisive è stata bassa. Il $61 \%$ non vi ha fatto ricorso, ma il $14 \%$ vi ha preso parte intensamente, e il $26 \%$ vi ha partecipato almeno una volta. Dunque si evidenzia un maggior uso di questo mezzo

17 Il costo medio della campagna è di Lit. 21,8 milioni. Scomponendo il dato in relazione alla esistenza di precedenti esperienze politiche, scopriamo che $\mathrm{i}$ «nuovi» alla politica hanno speso in media molto meno degli ex politici di professione: solo $17 \mathrm{mi}$ lioni contro i 27,5 milioni spesi mediamente da questi ultimi. 
TAB. 12. Campagna elettorale: strumenti di propaganda utilizzati (\%)

\begin{tabular}{lcc}
\hline Strumento & Utilizzato «molto» & $\begin{array}{c}\text { Utilizzato «poco o } \\
\text { per nulla» }\end{array}$ \\
\hline Spot Tv & 3,8 & 85,6 \\
Spot radio & 1,9 & 91,9 \\
Partecipazione a trasmissioni radio-Tv & 13,8 & 60,6 \\
Inserzioni sui giornali & 4,4 & 71,9 \\
Lettere agli elettori & 46,9 & 27,5 \\
Porta a porta & 71,9 & 11,3 \\
Comizi & 24,4 & 41,9 \\
Incontri con categorie e gruppi & 64,4 & 16,3 \\
\hline
\end{tabular}

meno d'immagine rispetto allo spot. $\mathrm{Ci}$ sono poi le lettere agli elettori, uno strumento, quello del mailing, meno costoso dei precedenti. Gli eletti di Forza Italia nel $47 \%$ dei casi hanno utilizzato molto questo strumento di propaganda, cercando il contatto personale con l'elettore. Tuttavia resta pur sempre quasi un terzo degli eletti che non lo ha utilizzato per nulla. Un altro mezzo di propaganda classico, il porta a porta, è stato usato da più di due terzi degli eletti. Soltanto 1 ' $11 \%$ non l'ha mai utilizzato. I comizi invece, strumento di propaganda da partito di massa ideologico per eccellenza, sono stati più ostici per i candidati di Forza Italia, al punto che solo un quarto di essi vi ha fatto ricorso intensamente, mentre quasi la metà non ne ha mai tenuti. Gli incontri con le categorie socio-economiche locali rappresentano, a differenza dei comizi di piazza, un mezzo che ci aspetteremmo essere tra i più usati dagli esponenti di Forza Italia. Ed in effetti, vi fanno ricorso $2 / 3$ degli intervistati. Sappiamo però che l'interesse del candidato non è stato ricambiato in modo forte dalle categorie, le quali pur essendo state disponibili all'ascolto, non si sono mostrate altrettanto generose nel sostegno materiale al candidato.

Possiamo a questo punto iniziare a formulare delle ipotesi sul tipo di classe politica che si sta formando a livello periferico. Essa non sembra volersi o potersi servire né degli strumenti mediatico-demagogici prevalenti a livello nazionale come gli spot, né di quelli tradizionalmente ideologico-partitici come i comizi. Predilige invece un approccio personale e pragmatico, basato sullo sfruttamento di legami amicali o professionali, e sulla ricerca di un rapporto personale con gli elettori e con le categorie economiche. L'ampio uso di strumenti quali il porta a porta e gli incontri con le categorie indica la predilezione per 
una forma di rappresentanza post-ideologica e post-partitica, economico-privatistica ma anche potenzialmente clientelare. I candidati si sono proposti in maniera individualistica, privi del sostegno forte di un apparato di partito, che globalmente è risultato indifferente alla loro sorte elettorale. Forza Italia non ha fornito aiuti finanziari o strumentali alla periferia, né vi è stata una mobilitazione della base sociale attraverso i club, che anzi sono stati percepiti come inerti o inefficaci. Le associazioni di categoria, pur essendo state uno degli obiettivi principali dello sforzo propagandistico dei candidati, non si sono lasciate coinvolgere in forme di sostegno diretto, assumendo un atteggiamento attendistico e prudente. Dal punto di vista organizzativo ne deriva l'immagine di un gruppo di amministratori locali poco coeso, che non ha sviluppato - in un momento intenso e quindi propizio al rafforzamento dei legami organizzativi come quello della campagna elettorale - una lealtà forte nei confronti del partito, e dunque difficilmente potrà fornire la base per un suo eventuale consolidamento organizzativo.

La mancata sponsorship di Forza Italia è particolarmente sentita dal consistente gruppo di ex professionisti della politica. Pur avendo ricevuto in media più contributi finanziari dal partito, essi hanno una percezione negativa del suo sostegno, sia come partito che come base di simpatizzanti. Hanno evidentemente maggiori difficoltà ad inserirsi in un contesto poco strutturato quale è quello di Forza Italia. La loro insoddisfazione nei confronti del partito è controbilanciata dal maggior appoggio percepito da parte degli amici, del comitato elettorale e delle categorie, oltre che della stampa locale, a riprova di una certa disponibilità di risorse e capacità politico-organizzative derivanti dalla propria esperienza politica antecedente, che li porta anche a una maggiore intensità nell'uso di un ampio ventaglio di strumenti di propaganda elettorale. Questi eletti sono più attivi e meglio inseriti nel proprio contesto sociale, ma percepiscono il partito come meno efficace nel ruolo di sostenitore della propria candidatura, e come vedremo più avanti sono anche $\mathrm{i}$ meno soddisfatti della sua realtà organizzativa.

\section{L'eletto e il partito: l'integrazione mancata}

Nella terza e ultima parte di questo articolo cercheremo di far emergere il grado di soddisfazione dell'eletto rispetto al par- 
tito, specificando il senso di utilità, efficacia e coesione degli eletti locali, e il loro grado complessivo di integrazione nella vita del partito. Dopo l'elezione, gli amministratori locali si trovano a far parte al contempo delle istituzioni e del partito. $\mathrm{Ci}$ chiediamo se in essi stia avvenendo un processo di identificazione con l'organizzazione, e se essi stiano sviluppando un senso di coesione in quanto gruppo di amministratori locali. Per un partito di eletti, è essenziale che nasca un esprit de corps tale per cui gli amministratori si presentino come gruppo compatto e coerente.

Per enucleare il livello di integrazione degli eletti in Forza Italia abbiamo rivolto loro una domanda sui temi discussi nelle riunioni del partito. $\mathrm{Ci}$ interessava sapere se si discute più di temi nazionali o locali piuttosto che di problemi organizzativi o di linea politica, e se si affronta il tema della raccolta di fondi. Questo per verificare il grado di socializzazione politica, e dunque integrazione complessiva degli eletti (tab. 13). I problemi locali vengono discussi nell'ambito del partito dall' $83 \%$ degli eletti, e questo è per certi versi ovvio, dato che si tratta dei problemi di più immediata competenza dei nostri intervistati. A sorprendere è semmai quel $17 \%$ che non ne parla mai, denotando in quasi un quinto del campione uno scarso interesse a discutere delle questioni di rilevanza politica locale nell'ambito del partito. Della linea politica più generale di Forza Italia invece non parla mai o solo raramente il $40 \%$, spesso solo il $20 \%$, abbastanza spesso il $40 \%$ degli intervistati. Sembrerebbe esserci, da parte di una fetta consistente di eletti, uno scarso interesse per le questioni politiche, specie se generali o nazionali. Questi risultati possono sembrare in linea con la realtà di un partito programmaticamente non ideologico, ma riflettono soprattutto l'esistenza della già rilevata cesura tra livello nazionale e livello locale dell'organizzazione, la quale trova ulteriore conferma in quel quarto di eletti che dichiara di non parlare mai o solo raramente delle tematiche relative all'organizzazione locale di Forza Italia. In un partito in cui è appena stato approvato un nuovo statuto e per il quale si sta rendendo operativa una nuova struttura con ripercussioni anche ai livelli organizzativi sub-nazionali è singolare che un quarto degli eletti se ne disinteressi completamente. $\mathrm{Ma}$ ancora maggiore è il numero di coloro che non affrontano mai il tema, apparentemente di rilevanza primaria, dell'organizzazione nazionale del partito. Ben $2 / 3$ degli eletti non ne parlano mai, mentre ne parla spesso solo 1 ' $11 \%$. Nemmeno la ricerca di 
TAB. 13. Temi discussi nelle riunioni degli organi locali di Forza Italia (\%)

\begin{tabular}{lc}
\hline Frequenza & Mai/raramente \\
\hline Temi: & \\
Problemi locali & 17,2 \\
Linea politica & 39,2 \\
Organizzazione locale & 25,0 \\
Organizzazione nazionale & 65,4 \\
Raccolta fondi & 65,6 \\
\hline
\end{tabular}

finanziamenti sembra essere un tema «caldo» nelle articolazioni periferiche di Forza Italia. Quasi un terzo degli eletti non ne parla mai, lo affronta raramente il $40 \%$, spesso solo il $15 \%$.

Se da un lato l'interesse degli eletti sembra concentrarsi sui problemi locali, e dunque sugli impegni derivanti dalla propria carica amministrativa, dall'altro lato emerge un apparente disinteresse per le questioni propriamente partitiche, come la linea politica, o i problemi organizzativi e finanziari. Allo scarso interesse del partito verso i propri candidati durante la campagna elettorale fa riscontro l'atteggiamento apparentemente disinteressato degli eletti nei confronti dell'organizzazione. Emerge dunque una reciprocità tra offerta e domanda, tra incentivi e livello di partecipazione. Il disinteresse del partito favorisce un atteggiamento non curante da parte dell'eletto. Che vi sia reciprocità non significa però che si sia raggiunto un punto di equilibrio. Al contrario, l'atteggiamento dell'eletto è il sintomo di una condizione di disagio, derivante come vedremo dalla percezione negativa del partito, e dalla sfiducia circa la possibilità di ottenere i cambiamenti ritenuti indispensabili.

Una riprova della scarsa integrazione e coesione organizzativa proviene indirettamente dalle risposte alla domanda relativa alla persona a cui l'eletto si rivolge quando deve risolvere questioni politiche controverse (tab. 14). Nella ricerca di soluzioni il contatto che l'eletto persegue è in un terzo dei casi quello con i rappresentanti del partito a livello locale, il coordinatore regionale e quello provinciale o cittadino. Il contatto con i livelli nazionali del partito è molto esiguo, ed è quasi inesistente quello con i rappresentanti dei club. Non c'è insomma un'integrazione verticale, non ci sono correnti, e nemmeno emergono delle tendenze, cioè dei legami verticali più deboli, ma comunque cementanti. Per altro, non vi è nemmeno una qualche integrazione orizzontale, come mostra non soltanto la scarsa percen- 
TAB. 14. Referente privilegiato nel partito (\%)

\begin{tabular}{lr}
\hline Referente & $\%$ \\
\hline Coordinatore regionale & 17,9 \\
Coordinatore provinciale & 10,7 \\
Capogruppo consiliare & 3,6 \\
Colleghi consiglieri & 11,4 \\
Parlamentare della sua regione & 5,7 \\
Delegato di collegio & 4,3 \\
Presidenti di club & 0,7 \\
Consiglieri regionali & 0,7 \\
Nessuno & 7,9 \\
Altri & 37,1 \\
\hline
\end{tabular}

tuale di eletti che ha fiducia nei rappresentanti locali del partito, ma anche l'esiguo numero di coloro che si rivolgono a colleghi consiglieri o al capogruppo consiliare. Non si sta dunque formando un'élite autonoma e integrata, autosufficiente dalle gerarchie di partito.

Una terza domanda verteva sull'intensità dei rapporti con i diversi dirigenti del movimento. Ebbene, solo poco più di 1/5 degli intervistati dichiara di avere rapporti stretti con i parlamentari della propria regione, mentre quasi la metà non ne ha nessuno o ne ha di occasionali (tab. 15). Inoltre, la quasi totalità del campione non ha contatti con parlamentari di altre regioni o con i membri del Comitato di Presidenza, organismo di cui fa parte la leadership del partito. Persino con il coordinatore regionale vi è una scarsa intensità di rapporti per più della metà degli intervistati, e rapporti intensi solo per poco meno di un terzo di essi. Con i presidenti di club i rapporti sono scarsi per metà degli intervistati, e 3/4 di essi dichiarano di non avere alcun rapporto con dirigenti del partito diversi da quelli contenuti nella domanda. Ne risulta l'immagine di un partito i cui livelli orizzontali sono rigidamente stratificati su base regionale, e poco integrati tra loro.

Gli eletti con precedenti esperienze politiche si distinguono dagli altri. Infatti, hanno rapporti più stretti con i parlamentari e con lo stesso coordinatore regionale: la percentuale di coloro che non hanno alcun rapporto col coordinatore regionale scende di una decina di punti percentuali. Questo avvalora i dati relativi al reclutamento e al finanziamento, che mostravano come gli ex fossero stati reclutati in maggioranza attivamente dal partito, attraverso un canale più gerarchico e finanziati in misura 
TAB. 15. Intensità di rapporti con dirigenti di Forza Italia (\%)

Intensità di rapporti

Nessuno/occasionali

Dirigente:

Parlamentare della sua regione

48,8

Membri comitato di presidenza

95,6

Coordinatore regionale

52,5

Presidenti di club

46,9

Altri dirigenti

76,9

maggiore rispetto agli altri, e come fossero meglio inseriti nel proprio contesto sociale. Ma ciò non significa che essi siano anche più integrati o soddisfatti - non a caso, sono anche quelli che hanno rapporti ancor meno stretti dei loro colleghi con $\mathrm{i}$ club, i quali come abbiamo visto precedentemente hanno fornito loro uno scarsissimo sostegno in campagna elettorale.

Per concludere questo primo tentativo di ricognizione tra gli amministratori locali di Forza Italia non ci restano da analizzare che i risultati relativi alle domande che misurano in maniera più specifica la soddisfazione degli eletti rispetto all'organizzazione.

Globalmente, più di $4 / 5$ degli intervistati ritengono che l'organizzazione vada cambiata $(84,8 \%)$. Anche in questo caso la percentuale di insoddisfatti è più alta tra gli eletti con precedente esperienza $(89,2 \%)$ che tra quelli «nuovi» alla politica $(81 \%)$.

Per quanto riguarda il tipo di modifiche da apportare all'organizzazione per migliorarla, nel questionario abbiamo presentato agli intervistati una serie di items legati al dibattito in corso nel partito nell'anno pre-congressuale, che aveva visto l'elaborazione e la presentazione di un nuovo statuto organizzativo e la convocazione di congressi provinciali in previsione del Congresso nazionale il 18 aprile 1998. Tuttavia, essendo al momento del sondaggio l'attuazione del nuovo statuto ancora lungi dall'essere compiuta, il giudizio degli eletti poteva risultare frutto dell'esperienza accumulata negli anni precedenti all'entrata in vigore dello statuto, e non implicava necessariamente un giudizio sul nuovo statuto. Per questo si è reso necessario formulare una ulteriore domanda relativa al grado di approvazione del nuovo statuto, che avrebbe consentito di misurare la percezione dell'eletto circa il futuro organizzativo del partito.

L'atteggiamento nei confronti delle riforme organizzative è assai favorevole. Le modifiche suggerite riscuotono un vasto consenso, a partire da un più forte radicamento locale per pas- 
TAB. 16. Tipo di modifiche organizzative auspicate (\%)

\begin{tabular}{lc}
\hline FI ha bisogno di: & Molto \\
\hline Più collegamento club/partito & 60,7 \\
Più sedi locali & 59,9 \\
Più club & 26,4 \\
Più funzionari & 63,4 \\
Elezioni organi dirigenti locali & 85,3 \\
Elezioni organi dirigenti nazionali & 74,3 \\
Più dibattito sulla linea politica & 79,9 \\
Congresso nazionale & 81,9 \\
Congressi regionali & 82,7 \\
Meno conflittualità a livello locale & 76,5 \\
Meno conflittualità a livello nazionale & 49,7 \\
Più contatti personali & 81,5 \\
Più considerazione esigenze locali & 88,8 \\
Più collegamento tra livello loc. e naz. & 88,7 \\
Elezioni primarie & 53,3 \\
\hline
\end{tabular}

N.B. Il questionario prevedeva la possibilità di indicare più di una risposta.

sare a una maggiore interazione, collegamento, coesione e coerenza strutturale. Inoltre, sono viste molto positivamente anche eventuali elezioni degli organi dirigenti locali e nazionali, così come molto sentita è l'esigenza di un congresso nazionale e di congressi regionali. Più bassa è invece la percentuale di chi ritiene necessarie elezioni primarie per la scelta dei candidati mentre quasi $3 / 5$ degli intervistati dicono di no a un incremento del numero di club (tab. 16).

Gli ex politici anche in questo caso hanno atteggiamenti più marcatamente critici, anche se in maniera differenziata. Sono favorevoli ad una maggior capillarità sul territorio, ma anche al mantenimento di una struttura leggera, non burocratica. Ritengono in misura maggiore che $\mathrm{i}$ club vadano meglio integrati nel partito, che ci voglia più discussione della linea politica, un maggior sviluppo di contatti personali e più collegamento tra livello locale e nazionale. Inoltre, essi ritengono più necessaria l'elezione degli organi dirigenti locali e nazionali, così come ancor più sentita è l'esigenza di un congresso nazionale e di congressi regionali.

Queste cifre ci hanno dato indicazioni importanti sulla soddisfazione degli eletti rispetto alla struttura e al suo funzionamento, confermate dalla valutazione del nuovo statuto. Solo il $3,4 \%$ del nostro campione lo valuta molto positivamente, ritenendolo in grado di porre rimedio alle insufficienze organizzati- 
ve del partito. Poco meno di un quinto lo valuta abbastanza positivamente; il 40,7\% degli eletti lo ritiene sufficiente; quasi $1 / 3$ di essi lo giudica non soddisfacente. Gli ex professionisti della politica ne hanno un'opinione più negativa rispetto al resto del campione.

In definitiva, possiamo concludere che, sia il senso di utilità che l'integrazione si sono rivelati piuttosto bassi. Gli eletti tendono a disinteressarsi alle questioni sia organizzative sia di linea politica, reputando l'organizzazione del partito estremamente carente e poco idonea a fornire loro gli incentivi attesi in cambio della propria partecipazione. Il partito è percepito dai più come un'entità estranea, che necessiterebbe di profondi cambiamenti, rispetto al verificarsi dei quali tuttavia la fiducia è bassa. L'integrazione sia verticale che orizzontale risulta assai limitata. Non sembra pertanto in via di formazione un'élite coe$\mathrm{sa}$, indipendente e in grado di sfruttare risorse autonome. I rapporti interni alla subunità organizzativa costituita dagli eletti sono poco intensi e articolati. Gli ex professionisti della politi$\mathrm{ca}$, pur maggiormente integrati nel partito, ne sono anche maggiormente insoddisfatti, e dunque ancor meno disponibili a dirigere la propria lealtà verso l'organizzazione. In generale, il desiderio di profonde riforme strutturali che vanno in direzione di un partito più presente a sostegno dei propri rappresentanti nelle istituzioni locali si scontra con la scarsa fiducia nella capacità del nuovo statuto organizzativo di porre rimedio alle gravi insufficienze riscontrate dagli eletti.

\section{Conclusioni}

In questo articolo si è cercato di individuare le possibilità di persistenza organizzativa e di istituzionalizzazione di Forza Italia. Si è ritenuto che la capacità da parte di questa nuova forza politica di radicarsi localmente passi per necessità, data la struttura «leggera» e non burocratica da partito degli eletti che ha voluto darsi, attraverso il legame che essa è in grado di instaurare con i propri rappresentanti locali e la coesione che si realizza tra di essi. In una organizzazione di questo tipo infatti deve instaurarsi un legame forte tra gli eletti, in quanto essi costituiscono all'interno e rappresentano all'esterno l'elemento portante e distintivo dell'organizzazione. L'infusione di valori, passaggio fondamentale del processo di istituzionalizzazione, 
passa inoltre attraverso la creazione di un legame tra l'eletto e l'organizzazione, tale da permettere al primo di identificarsi con la seconda indipendentemente dalla capacità di quest'ultima di perseguire efficacemente gli scopi ufficiali. Se è difficile che ciò avvenga per gli eletti al parlamento, in quanto essi sono legati strettamente alla fonte della legittimazione organizzativa verso cui tutta la lealtà si indirizza, ovvero la sua leadership patrimoniale (Maraffi 1995), ha tuttavia senso chiedersi se gli amministratori locali si trovino nella stessa condizione, o invece essi possano e stiano di fatto sviluppando un interesse più stabile $\mathrm{e}$ autonomo alla sopravvivenza organizzativa. Questo perché la loro lealtá nei confronti di Forza Italia sarebbe mediata solo in forma debole dalla lealtà personale verso il leader. Ma affinché tale interesse alla sopravvivenza dell'organizzazione si manifesti, occorre che emerga una complementarietà tra le motivazioni individuali degli eletti e gli incentivi loro offerti da parte dell'organizzazione. Questo articolo ha cercato di verificare se tale complementarietà si sia realizzata o meno. Abbiamo indagato il rapporto di scambio tra gli eletti e il partito, focalizzando in modo particolare l'attenzione sulle tappe attraverso le quali si consolida il rapporto tra eletto e organizzazione: il reclutamento, la campagna elettorale e il periodo infra-elettorale. Ma né le modalità di reclutamento e selezione dei candidati, né il comportamento del partito in campagna elettorale, né le dinamiche organizzative del periodo successivo all'elezione hanno posto delle basi favorevoli all'infusione di valori, e di conseguenza al consolidamento e alla istituzionalizzazione di Forza Italia, quanto meno a livello locale. Permanendo questo stato di cose, difficilmente gli eletti locali potranno trasformarsi in un gruppo coeso e identificato, in grado di opporsi e resistere alla possibilità di dissolvimento dell'organizzazione, sempre attuale a causa del suo status di partito personalistico e patrimoniale. Per lo stesso motivo, d'altro canto, essi non potranno costituire un vivaio a cui attingere per rinnovare la classe dirigente nazionale in una direzione più autonoma della leadership. Di conseguenza, le prospettive generali di istituzionalizzazione di Forza Italia restano molto basse. La strategia del franchising può essere stata funzionale al mantenimento temporaneo di una struttura monocratica, ma non lo è certo ai fini del reinserimento stabile nel circuito della partecipazione politica di tutti coloro che al principio degli anni novanta sono rimasti privi di un referente politico-partitico (Morlino 1996; Bardi e Morlino 1994). Permanen- 
do questa configurazione organizzativa di Forza Italia, un consolidamento del centro-destra potrà forse anche realizzarsi, ma è destinato a mantenere un carattere sostanzialmente precario.

\section{Riferimenti bibliografici}

Baget Bozzo, G. (1998), La cultura politica di Forza Italia: il liberalismo popolare, opuscolo distribuito in occasione del Congresso di Milano del 18/4/98, Roma, Struttura Formazione di Forza Italia.

Bardi, L. e L. Morlino (1994), Tracing the Roots of the Great Transformation, in R. Katz e P. Mair (a cura di), How Parties Organise, London, Sage, pp. 242-277.

Bartolini, S. e R. D'Alimonte (1997), Il maggioritario dei miracoli, in R. D'Alimonte e S. Bartolini (a cura di), Maggioritario per caso. Le elezioni politiche del 1996, Bologna, Il Mulino, pp. 351-370.

Berlusconi, S. (1998), Introduzione, in Baget Bozzo (1998), pp. 7-11.

Clark, P.B. e J.Q. Wilson (1961), Incentive Systems: a Theory of Organisations, in «Administrative Science Quarterly», n. 4, pp. 129-166.

Dalton, R., S. Flanagan e P.A. Beck (a cura di) (1984), Electoral Change in Advanced Industrial Democracies. Realignment or Dealignment?, Princeton, Princeton University Press.

Ferrante, M. (1998), Transizione di regime e interessi imprenditoriali in Italia, in «Rivista Italiana di Scienza Politica», n. 1, pp. 81-116.

Gallagher, M. e M. Marsh (a cura di) (1988), Candidate Selection in Comparative Perspective. The Secret Garden of Politics, London, Sage.

Hopkin, J. e C. Paolucci (1999), The Business Firm Model of Party Organisation. Cases from Spain and Italy, in «European Journal of Political Research», n. 3, pp. 307-339.

Huntington, S. (1968), Political Order in Changing Societies, New Haven, Yale University Press.

Kriesi, H. (1998), Il cambiamento dei cleavages politici in Europa, in «Rivista Italiana di Scienza Politica», n. 1, pp. 55-80.

Maraffi, M. (1995), Forza Italia, in G. Pasquino (a cura di), La politica italiana. Dizionario critico 1945-1995, Roma-Bari, Laterza, pp. 247-259.

- (1996), Forza Italia dal governo all'opposizione, in M. Caciagli e D. I. Kertzer (a cura di), Politica in Italia. I fatti dell'anno e le interpretazioni. Edizione '96, Bologna, Il Mulino.

McAllister, J. (1997), Australia, in P. Norris (a cura di), Passages to Power. Legislative Recruitment in Advanced Democracies, Cambridge, Cambridge University Press, pp. 15-32.

Morlino, L. (a cura di) (1991), Costruire la democrazia, Bologna, Il Mulino. 
- (1996), Crisis of Parties and Change of Party System in Italy, in «Party Politics», n. 1, pp. 5-30.

Norris, P. (1997), Introduction. Theories of Recruitment, in Id. (a cura di), Passages to Power. Legislative Recruitment in Advanced Democracies, Cambridge, Cambridge University Press.

Norris, P. e J. Lovenduski (1997), United Kingdom, in P. Norris (a cura di), Passages to Power. Legislative Recruitment in Advanced Democracies, Cambridge, Cambridge University Press, pp. 158186.

Panebianco, A. (1982), Modelli di partito. Organizzazione e potere nei partiti politici, Bologna, Il Mulino.

- (1995), Tre mosse necessarie per un vero partito, in «Liberal», n. 4, luglio.

Pizzorno, A. (1993), Le difficoltà del consociativismo, in Id., Le radici della politica assoluta e altri saggi, Milano, Feltrinelli.

Poli, E. (1997), I modelli organizzativi, in D. Mennitti (a cura di), Forza Italia. Radiografia di un evento, Roma, Ideazione Editrice, pp. 79-109.

Porro, N. (1995), L'innovazione conservatrice. Fininvest, Milan club e Forza Italia, in «Quaderni di Sociologia», n. 9.

Pozzi, R. e S. Rattazzi (1994), Farsi eleggere. La campagna elettorale nella Seconda Repubblica, Milano, Il Sole 24-Ore Edizioni.

Putnam, R.D. (1976), The Comparative Study of Political Élite, Englewood Cliffs, N.J., Prentice Hall, Inc.

Roback,T.H. (1974), Recruitment and Incentive Patterns among Grassroots Republican Officials: Continuity and Change in Two States, Beverly Hills-London, Sage.

Schlesinger, J.A. (1984), On the Theory of Party Organisation, in «The Journal of Politics», n. 46, pp. 369-400.

Selznick, P. (1957), Leadership in Administration, New York, Harper \& Row.

Sofauf, F.J. (1968), Party Politics in America, Boston, Little Brown and Co.

Tonarelli, A. (1999), Gli amministratori locali di Forza Italia. Modelli di reclutamento e di rappresentanza sociale, in «Rivista Italiana di Scienza Politica», n. 1, pp. 89-119.

Verzichelli, L. (1997a), La classe politica della transizione, in R. D'Alimonte e S. Bartolini (a cura di), Maggioritario per caso, Bologna, Il Mulino, pp. 309-350.

- (1997b), La classe parlamentare, in D. Mennitti (a cura di), Forza Italia. Radiografia di un evento, Roma, Ideazione Editrice, pp. 4977.

Weber, M. (1995), Economia e società, [vers. orig. Wirtschaft und Gesellschaft, 1922], vol. IV: Sociologia Politica, Milano, Edizioni di Comunità. 